\title{
How agency models inspire large scale participatory planning and its evaluation
}

\author{
Ferrand, N. ${ }^{a}$, Hassenforder, E. ${ }^{\text {ab }}$, Ducrot, R. ${ }^{c}$, Barreteau, O. ${ }^{a}$ and Abrami, G. ${ }^{a}$ \\ ${ }^{a}$ IRSTEA, G-EAU; ${ }^{b}$ Australian National University, ${ }^{c}$ CIRAD G-EAU \\ Email: nils.ferrand@irstea.fr
}

\begin{abstract}
We describe how three models, for sustainable change, human agency in collective resource management, and socio-environmental systems, have been used to design a protocol and the tools for a large scale (1500 participants, 35 villages) multi-level participatory process held in Africa for Integrated Natural Resource Management, through the European Project Afromaison. The process especially combines a common action model to support proposals by stakeholders, an integration matrix to build coherent plans, a role playing game design process, and a method to combine planning and playing to engage into the plans. It has also inspired the design of the attached monitoring and evaluation process. We describe the process in two countries, Ethiopia and Uganda, present the theoretical bases of the evaluation framework using the ENCORE paradigm and the implemented methodology transferred to local evaluators. We introduce some results and propose comments on potential learning back to the modelling community.
\end{abstract}

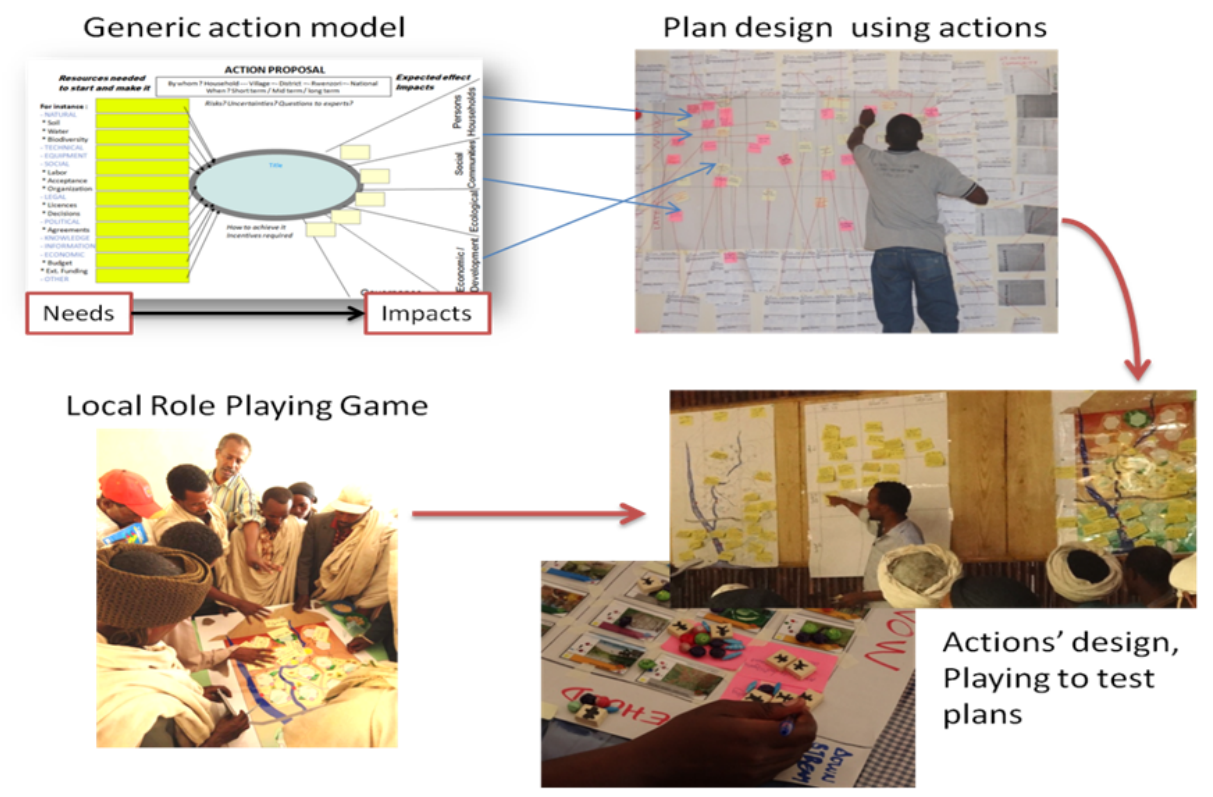

Figure 1. The main methodological tools

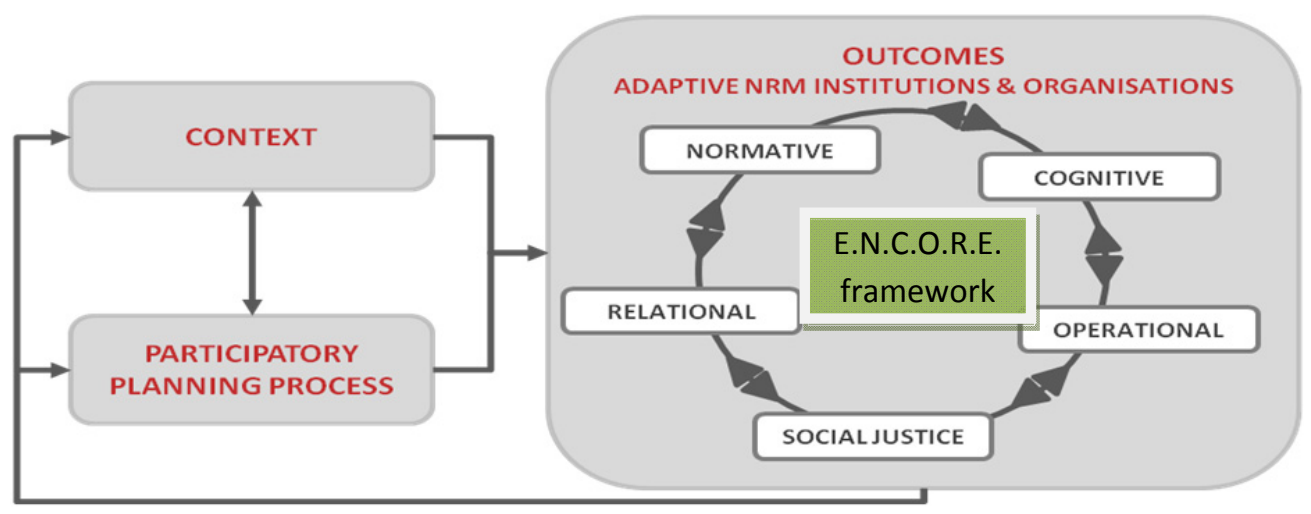

Figure 2. Theoretical Framework for the Monitoring \& Evaluation process

Keywords: Participatory planning, monitoring, evaluation, integrated natural resource management, roleplaying-game, Africa, multi-level governance 
Ferrand et al., How agency models inspire large scale participatory planning and its evaluation

\section{INTRODUCTION}

Integrated natural resource management (INRM) requires coping with diverse domains, issues, scales and stakeholders. Managers must design and implement processes and frameworks which can actually deal with this complexity for different terms, engage the local society, satisfy expectations and be implemented. Large scale participatory modelling is a natural candidate for such constrained challenge. In this paper we try to elicit how various frameworks inspired from agent based modelling and its applications have driven the design of the process and its evaluation.

The FP7 European Union funded project "AfroMaison" (2011-2014) aims at bringing the concept of integrated natural resources management (INRM) into practice by developing a dedicated toolbox and operational framework. One sub-part of it concerns the proposition and validation of concrete INRM plans. The challenge is to get to efficient plans actually engaging all stakeholders, coherent with the existing or emerging institutions, and finally implemented.

This common operational framework has been implemented in 5 countries (Tunisia, South Africa, Ethiopia, Uganda and Mali) by local partners. It intensively and directly engages multi-level stakeholders in assessing the situation (resources, well-being, drivers) and the envisioned evolutions in front of global change, in proposing and specifying various actions (natural resource use and management, regulation instruments, information, organization...), and structuring them in coherent multi-sectoral multi-level plans. These plans are explored and tested through dedicated role-playing games (RPG) locally designed by key actors to represent their issues and complex linkages between society and the environment. A continuous monitoring and evaluation protocol provides information about the process and its outcomes. The initial question is to which extent such process would lead to common visions, and induce plans appropriation and improvement, eventually leading to the emergence of meso-scale institutions implementing these plans.

Innovation in this research approach resides in several aspects:

1. The complex system is embraced as a whole: social interactions/the environment is not disconnected from the process and its outcomes;

2. It has a fully assumed action-research posture with a robust monitoring protocol, process-based, allowing to focus on efficiency indicators and to establish the distinction between process-driven and externallydriven outcomes;

3. Autonomy of local communities and partners in the process is favoured so that large scale social extension can be locally managed and supported in the long term. Direct foreign intervention is minimized.

4. The design of the game is expert-based with multiple inputs from "close friends" and meso-level stakeholders for readjusting and simplifying the game components and dynamics 'on the way';

5. Exploration and testing of the plans through self-built role-playing games is an innovation per se;

6. Monitoring and evaluation is also as much as possible transferred to the local groups.

7. The playing and monitoring processes take place in and have to adapt to a low-tech context with loweducation levels (at the local level up to $50 \%$ of participants are illiterate).

Some of these aspects are broad research questions which even exceed the frame of agent-based modelling.

\section{GROUNDING AN INTERVENTION ON A MODEL OF AGENCY AND MULTI-LEVEL PLANNING}

This research project globally aims at improving community well-being in Africa by fostering new sustainable management of their natural resources, accounting for many dimensions. It should design and validate tools therefore. In this framework, we focus our contribution on a participatory process which should facilitate the integration of multiple levels, sectors, stakeholders, time-frames in an implementable strategy. Therefore we assume that large scale co-modelling of strategies and participatory simulation with games are relevant tools for these social and cognitive aims, reminding that multi-agent models and games have common conceptual origins (Barreteau \& al, 2001). The design and evaluation of this process is based on a reference model of socio-environmental system and agency for sustainable change. It grounds our theory of intervention. We follow a pretty classical paradigm inspired from the early user modelling thoughts (Sleeman et al., 1985). It aggregates: 
1. A model of sustainable change (and toward sustainability), which frames its own planning process: it assumes a classical needs / impact framework for, firstly, action assessment, and secondly, strategy evaluation. Actions engaged, either physical (infrastructures), technical (equipment) or human-based (information, learning, policy...), consume resources (material or immaterial) and induce multidimensional outcomes. These outcomes can be evaluated against any multi-criteria position of stakeholders about sustainability. An action can recursively be defined as a set of others. The change strategy is supposed to be sustainable per se when three internal conditions are met: feasibility and coherency for accessibility to resources, no (uncompensable) antagonisms between outcomes and resource needs, compliance of the outcomes with the stated criteria by stakeholders. In the basic procedure, the complexity of links between actions and outcomes is processed via the diverse capacity of participants to deliberate and address this issue. A reference model of the environment is used to tackle the combined effect of different actions. This is done either using causal map of the environmental system (made initially by stakeholders and experts), or board simulation (i.e. role playing game), or computer based model. This leads to the participatory planning process which is presented later in this article.

2. A model of human agency elaborated as an extension of the classical Simon's (Simon, 1966) model of bounded rationality, through the Belief-Desire-Intentions paradigm, associated with assumptions on the impact of multiplex social networks (diverse relational types address cognition and norms), and a pragmatic dissociation acknowledged between the statement of intentions and their actuation. In this model we integrate a cognitive dimension, whereby people have beliefs or representations which can be formulated, structured and learnt -about themselves, their environment, others or the process-, a normative dimension, whereby people explicitly exhibit preferences or values which can be attached to knowledge, to social relationships and to practices, a relational dimension, whereby people have social links which differentially influence their beliefs and norms and have a coupled dynamic with the previous, an operational dimension, whereby intentions are split from actions, acknowledging contingencies, inconsistencies and non-intentional behaviours. Combined altogether, these dimensions lead to design processes with cognitive learning and structuring, normative exposure, framing and tagging (value attribution), dynamic linking between actors and procedural support therefore, commitment stating and real-world operations tracking. This model of agency frames also the vision of institutions which underlines the process design, as institutions are assumed to tackle (selectively) the various agency dimensions : learning (framing beliefs), normative change, establishing relations, constraining actions.

3. A simple stock-flow model of environment (context of the activities) based on a notion of resources (material or not) and exchanges, with specific interactions with the stakeholders. Such model is easily expressible and explored with stakeholders. It also fits the grounding model of the Wat-A-Game toolkit used in the processes (Abrami et al., 2012).

These 3 models taken together as reference and as empirical base for the participatory process design (for procedure and tools), is also used for designing the monitoring and evaluation protocol.

\section{THE PARTICIPATORY PLANNING PROCESS}

Two of the 5 case study areas are considered within the frame of this article: the Rwenzori region in western Uganda and the Fogera watershed flowing into the Tana Lake in Ethiopia.

The participatory planning process agreed with the initial steering group (meso-level group) contains 6 main phases. This group is composed of stakeholders having a connection with INRM or one sub-domain, and who come from different sectors: tourism, research, civil society, industry, agriculture, etc. We tried to balance conditions of representativeness in terms of gender, socio-economic and geographical backgrounds. Meso-scale is understood as an intermediary level between local (or community) level and national level. The operational definition of that scale as interpreted by the project teams corresponds to a range of mountains including a $200 \mathrm{~km}$-long catchment in Uganda $(13,970 \mathrm{~km} 2)$ and a subcatchment of the Blue Nile basin in Ethiopia (15,076 km2) (Migongo-Bake \& Catactutan, 2012).

The participatory planning process (adapted from the Aquastress integrated project (N. Ferrand, Hare, \& Rougier, 2006) includes 6 main phases:

1. Procedural agreement

2. Evaluation and identification of an initial long term common objective

3. Action proposal

4. Selection and integration of actions (planning)

5. Test of the plan using a participatory simulation tool (role playing game) 
Ferrand et al., How agency models inspire large scale participatory planning and its evaluation

\section{Implementation plan}

These steps along with their chronological order have been adapted by the project teams and stakeholders to the specificities of local institutional context and their on-going dynamics. For instance, social and political conditions in Uganda and Ethiopia led to select social extension, to assume some pre-existing plans as given, to manage various agenda, to engage more or less into implementation.

It is based upon a sequence of participatory sessions including stakeholders from grassroots level up to highest institutional levels, jointly or in parallel. The main methodological tools used are:

1. A generic action template used by all stakeholders (paper format). This action template fits to the model of sustainable change, and connects with the normative and operational part of the agency model. It brings users to specify and discuss the needs, the expected impacts, and the implementation pathway.

2. An integration protocol and a matrix to combine the actions into multi-level plans and discuss their coherence, feasibility and efficiency. Actions are organized in time, scales and space. Resources' needs and effects are measured qualitatively for deliberation. It's compatible with the model of the environment.

3. A local role-playing game conception process using the Wat-A-Game toolbox (Abrami et al., 2012; Nils Ferrand, Farolfi, Abrami, \& Du Toit, 2009), The meso-scale area of focus is represented through a game board whereby usual actions can be played, along with innovative actions coming from the plan. Players embody different roles corresponding to the major socio-economic groups in the region (Abrami et al., 2012). Roles and actions in the game are based on the agency model, and on the previous phases.

4. A protocol combining planning and gaming in order to explore, test and improve the plans.

This protocol integrates all elements and previous results. It uses a role-playing game for a "virtual test" procedure. The construction of the game has been made by the local team with the methodological support of experts. Subsequent modifications are added by "close friends" and other key stakeholders throughout sessions which repeatedly test the game, while also testing some plans. With some communities and social groups, the planning process was coupled with the role-playing game sessions. In Ethiopia for instance, the game board was used directly as a support for the spatial and temporal planning ; which in Uganda had been achieved previously with the integration matrix.

In Uganda, the participatory planning process was implemented in several steps with different social and political levels, engaging 35 communities (600 direct players and approximately 900 indirect observers). The game was used as a means to foster discussion and suggest innovative actions among local communities. Although 3 initial strategies had been proposed by a meso-scale group, all communities could also use the game to propose their own actions and finally share them. The same process was implemented at the district level with district leaders and at the national level with parliamentarians (vertical outscaling). The plan testing protocol was applied to the initial meso-scale set of actions, with the capacity given to communities to propose their own actions and test them, especially for groups who could play several sessions (up to 7). The 3 meso-scale strategies produced by the initial central steering group and the 27 strategies developed by the most active communities were integrated into a draft regional INRM strategy which feasibility and efficiency were discussed during a regional workshop held in early July 2013.

\section{MONITORING AND EVALUATION: CONCEPT AND PROTOCOL}

The monitoring and evaluation framework has to track from an external (observer) and an internal (subjective) position the situation in the sustainable change model (plan maturation), the agents' changes and the environmental effects, based on the three models. At the empirical level, within the AfroMaison project, an evaluation framework based on the "ENCORE" paradigm was suggested (Ducrot et al, 2013) for observing and qualifying the impacts of multi-level participatory processes, especially those with low disciplinary or paradigmatic bias or framing. "ENCORE" stands for 'External, Normative, Cognitive, Operational, Relational and Equity'. The framework aims at assessing the changes induced by a participatory process on these six dimensions (Ferrand, 2004 ; Ferrand \& Daniell, 2006) .

For implementing it, we start from classical criteria for the procedure and its effectiveness (Rowe, 2004). However, as we especially aim at assessing institutional and organizational change, and the plans' coherency, we also mobilize the work of authors on institutional and organizational dynamics (notably Ostrom, 2005, 2009; Saleth, 2006) as well as the literature on adaptiveness (Avenier, 2005; Pahl-Wostl, Kabat, \& Möltgen, 2008; Rijke et al., 2012; Simon, 1993) and robustness (Anderies, Janssen, \& Ostrom, 2004). We consider the notion of adaptive planning, often addressed in the context of climatic change (Grothmann \& Patt, 2005; Klein, Nicholls, \& Mimura, 1999). Between planning and adaptation, we also referred to adaptive or 
collaborative planning (Connick \& Innes, 2003; Hanak \& Lund, 2011; Lempert, Scheffran, \& Sprinz, 2009; Smith, 1973). With these grounds, we proposed an analytical framework for the 3 clusters: context, process and outcomes (Fig. 2), which has been derived into tools and procedures.

The main monitoring tool is a common "logbook" (Etienne, 2009) supposed to monitor all stakeholders interventions (timing), sessions, interactions, events, operational change, and other external or contextual factors (environmental, relational, socio-economic, discourse events). Attendance is tracked nominally, with literacy indicator. Participants fill simple "participants' evaluation sheets" for: satisfaction, learning (cognitive aspects), relational aspects, innovation/ creativity and commitment. For more intensive sessions, through an extended questionnaire they rate the process about access to information and expertise, representativeness, legitimacy / credibility, influence (expected real outcomes), transparency, timing, fairness in expression, convenience, and scale. After each session, facilitators fill in a "facilitator debriefing sheet" (open questions), to describe the process and its outcomes (cognitive, operational, relational aspects) including the action plan suggested. In Uganda, due to the wide extension of the process and our strongtransfer posture, this protocol is implemented autonomously by the stakeholders at the local scale, with all potential self-evaluation risks it may induce. This bias is limited by the conditioning of results and a triangulation of the data collected. Five "rapporteurs" were trained to monitor using a "Rapporteur debriefing sheet" based on participatory observation principles (feel, listen, observe) (Jankowski, 2012). It crosses similar variables and adds pictures of the session. Data analysis and comparison is shared between the local implementation group and the distant experts.

Due to contextual reasons, in Ethiopia, only part of these tools could be deployed. The logbook will only be filled in retroactively. For this meso-scale only process, "participants' evaluation sheets" were not required, and questionnaires have been adapted. Facilitator debriefing is made through direct discussion. Being more used to facilitation, the team was also keener in noting and reporting. Rapporteurs were not hired either since direct participatory observation could be made by the research team.

In parallel, semi-open interviews took place from august 2012 to july 2013 in Uganda to track evolution more qualitatively (a total of 31 participants and tiers like friends, relatives, plus process managers). In Ethiopia, 25 interviews of both participants and non-participants (including 3 focus groups of 3 people each) were made in April 2013. Both interviews were semi-open and based on a "life history" principle, asking about the AfroMaison process for participants and about other participatory planning processes for non-participants.

\section{FIRST CONCLUSIONS AND RECOMMENDATIONS}

This overall process had been designed with goals of increasing genericity: 1. propose and validate efficient INRM plans and actions, 2. assess this planning process, and its outcomes, including the emergence of new meso-scale institutions, 3. assess the tools used, 4. assess the overall intervention research of Afromaison, 5. assess the relevance of the grounding models. Our process-based monitoring and evaluation protocol aimed at this. At this stage the process and its evaluation is not yet finalized and we have collected preliminary results and comments for some of these dimensions. Detailed results will be provided after project's completion.

1. For the plans and actions, and for all case studies, some have been proposed, discussed, assessed, accepted, and in Uganda some parts of the plans have started being implemented. It's too early to address efficiency.

2. About the process and the tools used, and outside usual findings on power balance, inclusion and dynamic:

a. All steps and tools have actually been used by all levels of stakeholders. Everybody, individually or together could specify actions and plans descriptions (needs and outcomes), address coherency, and share them;

b. Various issues or dimensions of INRM could be explored and discussed by all participants;

c. The process has been really multi-scaled, both in terms of joint attendance, and combination of processes at different scales (in Uganda, from village to catchment)

d. Plans have been assessed both with the integration matrix by meso-scale groups and with the game by all groups

e. Most participants could publicly express commitments about actions and plans

f. The process, when open like in Uganda, has been highly attractive to people

3. About the process outcomes:

a. In cognitive terms, most participants expressed having learnt about the environment, and the complex interactions and consequences. They discover new practices and their integration. They also said (esp. Ethiopia) they have learnt planning skills. 
Ferrand et al., How agency models inspire large scale participatory planning and its evaluation

b. In relational terms, contacts have been established between groups, sub-regions and levels which are normally disconnected, even outside the involved participants. Mutual understanding was fostered (meso-scale people experienced more directly farmers' constraints).

c. In operational terms, some specific actions have been started in Ugandan villages (tree plantation, energy stoves, collecting plastic bags, etc).

d. In Uganda, some new environmental monitoring committees have been started

The design and evaluation process (Afromaison framework) is still to be evaluated in details, as it will be based also on ex-post scientists' interviews. But it's where the grounding models (sustainable change / agency / environmental dynamic) are the most challenged. In the multi-disciplinary context of this project, the research partners (scientists mainly) often disagree on the model of change drivers or pathway (engineers with technology vs. Biophysicists with structural change vs. Social scientists with human drivers) and agency (role of rationalized knowledge vs. Complex social processes). The focus put on data driven models (more accurate but time consuming) and technology transfer vs. co-design processes induces a different framework for INRM. Here we have chosen qualitative formalisms but high integration of issues, scales, etc. The key insight is that we have travelled from a set of concepts inspired from the agent models (computable), but haven't used any of these models for empirical reasons, and finally we support building empirical models which may feed into agent based modelling. For instance the characterization of actions by the stakeholders is a great source of information compatible with their actual agency processes. Additionally, the plans exhibited by stakeholders can be used by computer simulation designers as a grounded reference for design and for testing their models. A simulation which is not able to cope with such plans is not relevant for field needs. Finally the agency and institutions models can be strengthened by the combined observation of the participants and results of the subjective monitoring and evaluation.

\section{ACKNOWLEDGMENTS}

We acknowledge the financial support of the European Commission FP7 for the Afromaison project. This paper and the related results do not represent the view or engage any responsibility of the European Commission.

This process and results have been obtained thanks to (Uganda): Kabaseke, C., Muhumuza, M., Lemmens, R., Tibasima, T., Semana, A., Birungi, J., Kusemererwa, P., Mwanguhya, S., Akaganyira, S., Bwambale, G., Nzdghera, M., Mwesige Mujuku, R., (Ethiopia): Lemenih, M., Cullen, B., Lema, Z., Leta, G., Regassa, T., Molla, G., Zemadim, B., Desalegn, M., (France): Morardet, S., Baha, K., Gumpinger, E. (Belgium): Dhaeyer, T.

\section{REFERENCES}

Abrami, G., Ferrand, N., Morardet, S., Murgue, C., Popova, A., Fooij, H. De, ... Aquae-Gaudi, W. (2012). Wat-A-Game, a toolkit for building role-playing games about integrated water management. In D. B. (Eds. . R. Seppelt, A.A. Voinov, S. Lange (Ed.), International Environmental Modelling and Software Society (iEMSS) 2012 International Congress on Environmental Modelling and Software Managing Resources of a Limited Planet, Sixth Biennial Meeting. Leipzig, Germany. Retrieved from http://www.iemss.org/society/index.php/iemss-2012-proceedings

Anderies, J. M., Janssen, M. A., \& Ostrom, E. (2004). A Framework to Analyze the Robustness of Socialecological Systems from an Institutional Perspective. Ecology And Society, 9(1), 18. Retrieved from http://www.consecol.org/pub/www/Journal/Journal/vol9/iss1/art18/main.html

Avenier, M.-J. (2005). La Stratégie chemin faisant. In Cahiers de SOL (Society for Organizational Learning). Retrieved from http://halshs.archives-ouvertes.fr/halshs-00103059

Connick, S., \& Innes, J. E. (2003). Outcomes of Collaborative Water Policy Making: Applying Complexity Thinking to Evaluation. IURD Working Paper Series, Institute of Urban and Regional Development, UC Berkeley, 46(2), 177-197. doi:10.1080/0964056032000070987

Ducrot, R., Ferrand, N., Hassenforder, E., Gumpinger, E., 2013. Conditions and Results of a Joint Monitoring and Evaluation Protocol in 5 Countries. in proc. Science for the Environment conference 2013. Aarhus : PEER. http://dce-conference.au.dk/fileadmin/dce-conference.au.dk/abstracts/113 Ducrot.pdf

Etienne, M. (2009). La modélisation d'accompagnement: une démarche participative en appui au développement durable. (M. Etienne, Ed.) (Editions Q.). Paris, France.

Ferrand, N (2004). Participatory tools in the Water Framework Directive: what's for? Needs and requirements from HarmoniCop \& HarmoniQua. In Harmoni Concerted Action Conference - 2nd Catchmod technical workshop "Integrating tools and toolboxes." Copenhagen. Retrieved from http://www.harmoni-ca.info 
Ferrand et al., How agency models inspire large scale participatory planning and its evaluation

Ferrand, N., Hare, M., \& Rougier, J.-E. (2006). Iskar Test Site Option Description Living with Flood and Drought. Methodological document to the Iskar Test Site, AquaStress IP, FP6, Europe.

Ferrand, N, \& Daniell, K. (2006). Comment évaluer la contribution de la modélisation participative au développement durable? In Séminaire DDT. Lille.

Ferrand, N, Farolfi, S., Abrami, G., \& Du Toit, D. (2009). WAT-A-GAME: sharing water and policies in your own basin. In "Learn to Game, Game to Learn”, International Simulation And Gaming Association 40th Annual Conference. Singapore.

Grothmann, T., \& Patt, A. (2005). Adaptive capacity and human cognition: The process of individual adaptation to climate change. Global Environmental Change Part A, 15(3), 199-213. doi:10.1016/j.gloenvcha.2005.01.002

Hanak, E., \& Lund, J. R. (2011). Adapting California's water management to climate change. Climatic Change, 111(1), 17-44. doi:10.1007/s10584-011-0241-3

Jankowski, F. (2012). La Observación participante : Historia y principios.

Klein, R. J. T., Nicholls, R. J., \& Mimura, N. (1999). Coastal Adaptation to Climate Change: Can the IPCC Technical Guidelines be applied? Mitigation and Adaptation Strategies for Global Change, 4(3-4), 239-252. doi:10.1023/A:1009681207419

Lempert, R., Scheffran, J., \& Sprinz, D. F. (2009). Methods for Long-Term Environmental Policy Challenges. Retrieved from http://www.mitpressjournals.org/doi/abs/10.1162/glep.2009.9.3.106

Migongo-Bake, C., \& Catactutan, D. (2012). AfroMaison WP2 Report Chapter 6 Context analysis of Rwenzori mountains / Albertine Rift, Uganda.

Ostrom, E. (2005). Understanding Institutional Diversity. (I. University, Ed.)Public Choice (Vol. 132, p. 376). Princeton University Press. doi:10.1007/s11127-007-9157-X

Ostrom, E. (2009). A general framework for analyzing sustainability of social-ecological systems. Science (New York, N.Y.), 325(5939), 419-22. doi:10.1126/science.1172133

Pahl-Wostl, C., Kabat, P., \& Möltgen, J. (2008). Adaptive and Integrated Water Management: Coping with Complexity and Uncertainty. (C. Pahl-wostl, P. Kabat, \& J. Möoltgen, Eds.)October (Vol. 89, p. 306). Springer. doi:10.1029/2008EO330009

Rijke, J., Brown, R., Zevenbergen, C., Ashley, R., Farrelly, M., Morison, P., \& van Herk, S. (2012). Fit-forpurpose governance: A framework to make adaptive governance operational. Environmental Science \& Policy, 22(null), 73-84. doi:10.1016/j.envsci.2012.06.010

Rowe, G. (2004). Evaluating Public-Participation Exercises: A Research Agenda. Science Technology And Human Values, 29(4), 512-556. doi:10.1177/0162243903259197

Saleth, R. M. (2006). Understanding water institutions: Structure, Environment and Change Process. In Water Governance for Sustainable Development: Approaches and Lessons from Developing and Transitional countries (p. 320). Routledge.

Simon, H. A. (1966). Political research: the decision-making framework (David East.). Retrieved from http://doi.library.cmu.edu/10.1184/pmc/simon/box00084/fld06750/bd10001/doc0001

Simon, H. A. (1993). Strategy and organizational evolution. Strategic Management Journal, 14(S2), 131142. doi:10.1002/smj.4250141011

Sleeman, D., Appelt, D., Konolige, K., Rich, E., Sridharan, N. S., \& Swartout, B. (1985). User modelling panel. In Proceedings of the 9th international joint conference on Artificial intelligence (pp. 12981302).

Smith, R. W. (1973). A theoretical basis for participatory planning. Policy Sciences, 4(3), 275-295. doi:10.1007/BF01435125 